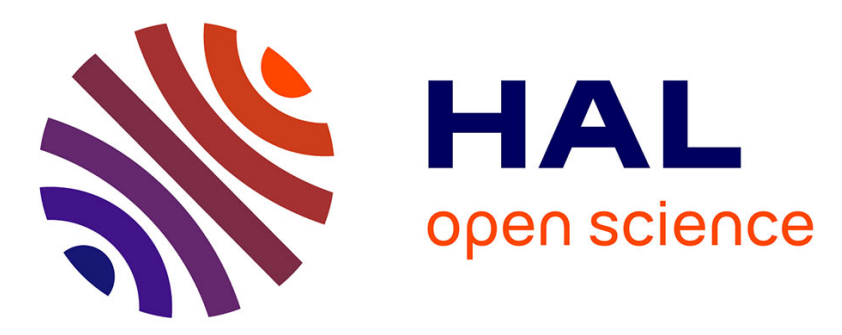

\title{
Next-generation telescopes with curved focal surface for ultra-low surface brightness surveys
}

Simona Lombardo, Eduard Muslimov, Gérard R Lemaitre, Emmanuel Hugot

\section{To cite this version:}

Simona Lombardo, Eduard Muslimov, Gérard R Lemaitre, Emmanuel Hugot. Next-generation telescopes with curved focal surface for ultra-low surface brightness surveys. Monthly Notices of the Royal Astronomical Society, 2019, 10.1093/mnras/stz2068 . hal-02263343

\section{HAL Id: hal-02263343 \\ https://hal.science/hal-02263343}

Submitted on 4 Aug 2019

HAL is a multi-disciplinary open access archive for the deposit and dissemination of scientific research documents, whether they are published or not. The documents may come from teaching and research institutions in France or abroad, or from public or private research centers.
L'archive ouverte pluridisciplinaire HAL, est destinée au dépôt et à la diffusion de documents scientifiques de niveau recherche, publiés ou non, émanant des établissements d'enseignement et de recherche français ou étrangers, des laboratoires publics ou privés. 


\title{
Next-generation telescopes with curved focal surface for ultra-low surface brightness surveys
}

\author{
Simona Lombardo, ${ }^{1 \star}$ Eduard Muslimov,${ }^{1}$ Gerard Lemaître,${ }^{1}$ and Emmanuel Hugot ${ }^{1}$ \\ ${ }^{1}$ Aix Marseille Univ, CNRS, CNES, LAM, Marseille, France
}

Accepted XXX. Received YYY; in original form ZZZ

\begin{abstract}
In spite of major advances in both ground- and space-based instrumentation, the ultralow-surface brightness universe (ULSB) still remains a largely unexplored volume in observational parameter space. ULSB observations provide unique constraints on a wide variety of objects, from the Zodiacal light all the way to the optical cosmological background radiation, through dust cirri, mass loss shells in giant stars, LSB galaxies and the intracluster light. These surface brightness levels (>28-29 mag arcsec $\left.{ }^{-2}\right)$ are observed by maximising the efficiency of the surveys and minimising or removing the systematics arising in the measurement of surface brightness. Based on full-system photon Monte Carlo simulations, we present here the performance of a ground-based telescope aimed at carrying out ULSB observations, with a curved focal surface design. Its off-axis optical design maximises the field of view while minimising the focal ratio. No lenses are used, as their multiple internal scatterings increase the wings of the point spread function (PSF), and the usual requirement of a flat focal plane is relaxed through the use of curved CCD detectors. The telescope has only one unavoidable single refractive surface, the cryostat window, and yet it delivers a PSF with ultra-compact wings, which allows the detection, for a given exposure time, of surface brightness levels nearly three orders of magnitude fainter than any other current telescope.
\end{abstract}

Key words: telescopes - instrumentation: detectors - surveys - cosmology: observations - galaxies: photometry - ISM: dust - stars: mass loss

\section{INTRODUCTION}

In the past decades the technological development has focused on improving the instruments and the techniques to test, e.g., the $\Lambda \mathrm{CDM}$ paradigm of galaxy formation and evolution on linear scales, such as the anisotropies of the Cosmic Microwave Background. However the non-linear scales still show some discrepancies between theoretical predictions and current observations and remain to be tested. One example is provided by the number of satellite galaxies (Bullock \& Boylan-Kolchin 2017) in the local group, which is order of magnitudes lower than what numerical simulations show. One of the reason for such difference might lay in the ultra-low surface brightness (ULSB) of these dwarf galaxies (well below the ground-based sky background), which would hence be missed by current surveys, not meant to observe very extended and faint structures.

Another discrepancy between prediction and observations can be found in the lack of an extended and irregular

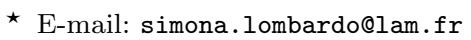

tidal tail around large galaxies which has been predicted (Kazantzidis et al. 2008; Cooper et al. 2010, 2013) but not significantly observed (Barton \& Thompson 1997; Fry et al. 1999; Atkinson et al. 2013).

Some progress has been made in the recent years and some of these features were observed (Martínez-Delgado et al. 2010; van Dokkum et al. 2015; Knapen \& Trujillo 2017; Mihos et al. 2017; Abraham \& van Dokkum 2014; Duc et al. 2015; Boissier et al. 2008). These surveys focus, however, on single objects or small regions in the sky, as they require long integration in order to reach the required signal-to-noise ratio (SNR), and a larger systematic study of the ultra-low surface brightness universe is still missing.

In order to design the ideal survey for this type of observations, one would choose: a place with the lowest skybackground level; a telescope without obscuration, as any obscuration or spider causes diffraction and displace more power in the point spread function (PSF) towards the wings (Singaravelu \& Cabanac 2014; Hugot et al. 2014; Muslimov et al. 2017a); a fast optical system with a small $f / D$ (where $f$ is the focal length and $D$ is the diameter of the entrance 
pupil), as for extended objects the imaging speed depends on the focal ratio (not on the aperture size, as for point-source objects); it should have either only refractive elements with anti-reflection coatings (e.g. Abraham \& van Dokkum 2014), or superpolished reflective surfaces, and no dust contamination.

A satellite with these characteristics would have the great advantage of drastically reducing the sky background and eliminating the molecular scattering due to the atmosphere (that degrades the PSF at large radial distances). The introduction of filter coatings directly deposited on the CCD surfaces would additionally allow to observe in several bands including UV (observable only from space). This is particularly important for observations of, e.g., the cosmic web filaments, believed to be brighter in UV wavelengths. The cosmic web of filaments is supposed to contain the missing fraction of baryons at low redshifts, therefore its observations would greatly improve our knowledge on the beginning and evolution of the universe.

This is the basic concept behind MESSIER (VallsGabaud \& MESSIER Collaboration 2017), a space mission that aims at measuring surface brightness levels as low as $35 \mathrm{mag} \operatorname{arcsec}^{-2}$ in the optical $(350-1000 \mathrm{~nm})$ and $38 \mathrm{mag}$ $\operatorname{arcsec}^{-2}$ in the UV $(200 \mathrm{~nm})$. As one of the goal is observing in the UV, all refractive elements must be avoided (as they will generate Cherenkov emission by the abundant relativistic particles) and the chosen design is therefore an unobscured fully reflective telescope with superpolished optics. The selected observation method is drift scan, which allows to achieve flat fielding accuracy of $0.0025 \%$ (Zaritsky et al. 1996). This additionally requires that the instrument distortion has to be corrected at least in one direction. The goals of the MESSIER mission are achievable with a high quality, $50 \mathrm{~cm}$ diameter, fast (f/2) UV-visible telescope with a field of view $>2^{o} \times 4^{o}$

Here we show a ground-based telescope (Section 2.1), that shares the main design concept of MESSIER and serves as a pathfinder and demonstrator to test and improve the curved sensor technology for astronomical usage. The design of the pathfinder was previously shown by Muslimov et al. (2017b) and presents a primary mirror of $35.6 \mathrm{~cm}$ with a field of view of $>1.6^{\circ} \times 2.6^{\circ}$. This telescope is a fully reflective Schmidt with a curved focal plane. The use of a curved CCD enhances the performances in terms of transmission and PSF shape, as it allows to simplify the overall system and (eliminates the need for field flattening lenses), while preserving the wide field of view (Rim et al. 2008; Blake et al. 2013; Guenter et al. 2017). Here we perform a series of photon Monte Carlo simulations (Section 3-4) to verify its performances also at very large distances from the centre of the field of view. We also simulate realistic observed fields and artificially inject some ULSB objects to test its full potential (Section 4.2).

We are hence able to demonstrate that the pathfinder is a competitor to all current ULSB surveys and, as it will be mostly used in drift-scan mode, it will allow to observe a large fraction of the sky every night, as opposite to the other surveys that are focusing on single objects.

\section{THE GROUND-BASED TELESCOPE DEMONSTRATOR}

In the next Section we will briefly describe the design of the pathfinder. Full scale simulations are required to asses the impact of possible scattered light and atmospheric effects over the full field of view of the telescope. As our goal is the observation of objects that are even fainter than a typical sky-background, the evaluation of the PSF, particularly at the wings, becomes a main parameter to explore. In this Section we also provide a description of the end-to-end photon Monte Carlo simulation software used.

\subsection{Telescope design}

The ground-based pathfinder is a fully reflective Schmidt telescope (Muslimov et al. 2017b), composed of an anamorphic primary mirror of $35.6 \mathrm{~cm}$ (whose purpose is to correct the spherical aberrations ), a flat secondary mirror and a spherical tertiary mirror that focuses the light onto a convex focal surface (Figure 1). The latter is equipped with a convex spherically-curved CCD with a radius of curvature of $\sim 900 \mathrm{~mm}$ and $4030 \times 2480$ pixels of $10 \mu \mathrm{m}$ pitch.

As we need to cool down and control the temperature of the CCD, the detector system is enclosed in a cryostat, whose window is the only, inevitable, refractive element of the system. The bandpass filters (one or two large-band filters) are deposited directly on the window, to optimize the performances. For simplicity we will only talk about a gband filter with the same characteristics as the one planned for the Large Synoptic Survey Telescope (LSST, Olivier et al. 2008), but more or different filters might be added in the future.

\subsection{Observing site}

Several dark sites are available in Europe for the commissioning and installation of this demonstrator. La Palma and Calar Alto are the best locations due to the quality of their skies (Leinert et al. 1995; García-Gil et al. 2010) and the accessibility of the observatory facilities. The choice of the site is particularly important to establish the main observing strategy, given the statistics of the atmospheric parameters. Both drift-scan and point-and-stare observing techniques are planned for the pathfinder, to allow for large sky coverage/per night (drift scan), or deeper observation of specific fields (point-and-stare) and critically depend on the darkness of the site. In this paper we will consider, as a proof of concept, a location within the Observatorio del Roque de los Muchachos in La Palma, for which there is a long record of atmospheric monitoring ${ }^{1}$.

\subsection{End-to-end Monte Carlo simulation software}

The software used to perform the end-to-end photon Monte Carlo simulations is PhoSim (Peterson et al. 2015) that allows to simulate photons from stars and galaxies, and raytrace them through the atmosphere, by creating seeing, atmospheric diffraction and extinction effects. Then the full

1 http://www.ing.iac.es/astronomy/observing/conditions/ 


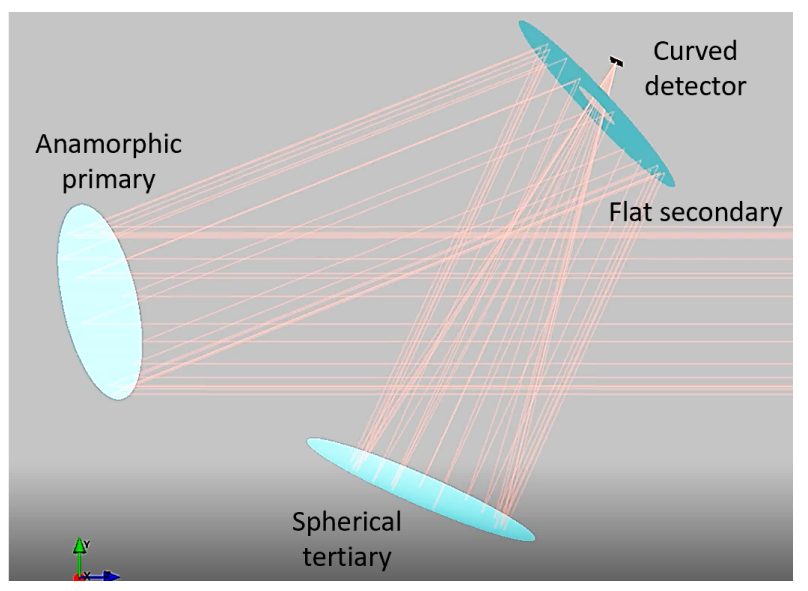

Figure 1. Optical design of the pathfinder: light reaches an anamorphic primary, is reflected by a flat secondary and a spherical tertiary yields a simple spherically-curved focal surface where a curved detector is placed.

optical system is realistically simulated including all the effects, such as misalignment, shifts, tilts and dust deposition on the optical surfaces. Finally the detector is simulated with all the effects due to charges diffusion in the silicon layer of the sensor and the noise errors due to readout, dark current, pixel response non-uniformity, etc.

Figure 2A shows the spot diagram in the focal plane of the telescope of a star simulated in the centre of the field of view. All the previously mentioned effects are added one after the other, to illustrate the capabilities of the software and the quality of the optical design. When simulating all the effects, most photons are still located within one pixel (10 $\mu \mathrm{m}$ pitch, corresponding to 2.32" angular dimension on sky) of the detector at the focal surface. Note that the "starshaped" image in the optical-design-only case in Figure 2A is due to the geometry of the distribution of rays in front of the entrance pupil of the telescope, and not to the presence of spiders holding the mirrors and obstructing the light. These elements were carefully avoided in the design of the pathfinder as they bring more power to the wings of the PSF. The only obstruction is due to the hole in the secondary mirror.

The quality of the PSF is ensured also at the edge of the field of view, as shown in Figure $2 \mathrm{~B}$ for a star observed at $0.7 \times 1.2$ from the centre of the field of view. The image appears more deformed than in the previous case, but most rays are still focused within a single pixel. The benefit of using a curved detector appears particularly evident at the edge of the field of view, where a better uniformity of the PSF is ensured with respect to the case where field flattening optics are used in combination with a flat detector (Muslimov et al. 2017b).

This is a key point to consider when the observing strategy includes the drift-scan. In this case the star is observed across the full field of view, and an highly distorted image at the corner of the field would result in more pronounced PSF wings. The uniformity of the PSF on the full field of view is hence a parameter to consider and optimize already at the optical design stage.

The reliability of the photon propagation in the simula-
Table 1. Site data and atmospheric parameters used for the simulations.

\begin{tabular}{lr} 
Site and mean atmospheric data \\
\hline & \\
Latitude [W] & $28^{\circ} .76$ \\
Longitude & $17^{\circ} .89$ \\
Altitude & $2326 \mathrm{~m}$ \\
Seeing & $1^{\prime \prime} 0$ \\
Dome seeing & $0^{\prime \prime} 1$ \\
Aerosol optical depth & 0.061 \\
Aerosol index & 0.85 \\
\hline
\end{tabular}

tion software was tested by comparing the spot diagrams at the focal surface of the telescope in the optical-design-only case with the equivalent simulation in ZEMAX. These two provided the same results, as already verified for the LSST case (Peterson et al. 2015, where it is also possible to find an accurate description of the physics involved in the simulation of the atmosphere).

\section{SIMULATION SETUP AND INPUT PARAMETERS}

PhoSim allows the user to modify the files used to set all the input parameters for the simulation. These files describe not only the optical design but also the observing site characteristics and location, which determine the atmospheric parameters. The input files describing typical atmospheric conditions have been prepared to accurately include the proposed observation site characteristics.

The wind speed and direction data, used to simulate the atmospheric turbulence for La Palma, are estimated from Kalnay et al. (1996). In Table 1 are indicated the location information and averaged values for some of the parameters. The seeing used is an intermediate value between the best $\left(0{ }^{\prime \prime} 72\right)$ and the worst $\left(1{ }^{\prime \prime} 3\right)$ seeing at La Palma observatories $^{2}$. The aerosol parameters are estimated from the averages of the year 2017 measured by AERONET aerosol robotic network ${ }^{3}$ for the Izaña location.

Other parameters, e.g. dome seeing, for which there are no known data, have the same values as provided for the LSST example in the code. In PhoSim the surfaces of the optical elements presents some microroughness which scatters light incident at large angles and consequently distribute more power to the wings of the instrument PSF. This feature is kept in the pathfinder simulations, so that we can study its performances and decide in a later stage if superpolished surfaces (where such microroughness effects will be strongly reduced) are necessary or can be avoided.

To add more realism in the simulations, the telescope surfaces are misaligned through shifts and tilts whose probability distribution is given by ZEMAX tolerances file. To reduce the number of refractive elements in the design, we

\footnotetext{
2 http://www.ing.iac.es/astronomy/observing/conditions/

3 http://aeronet.gsfc.nasa.gov/cgi-bin/draw_map_display_ aod_v3
} 


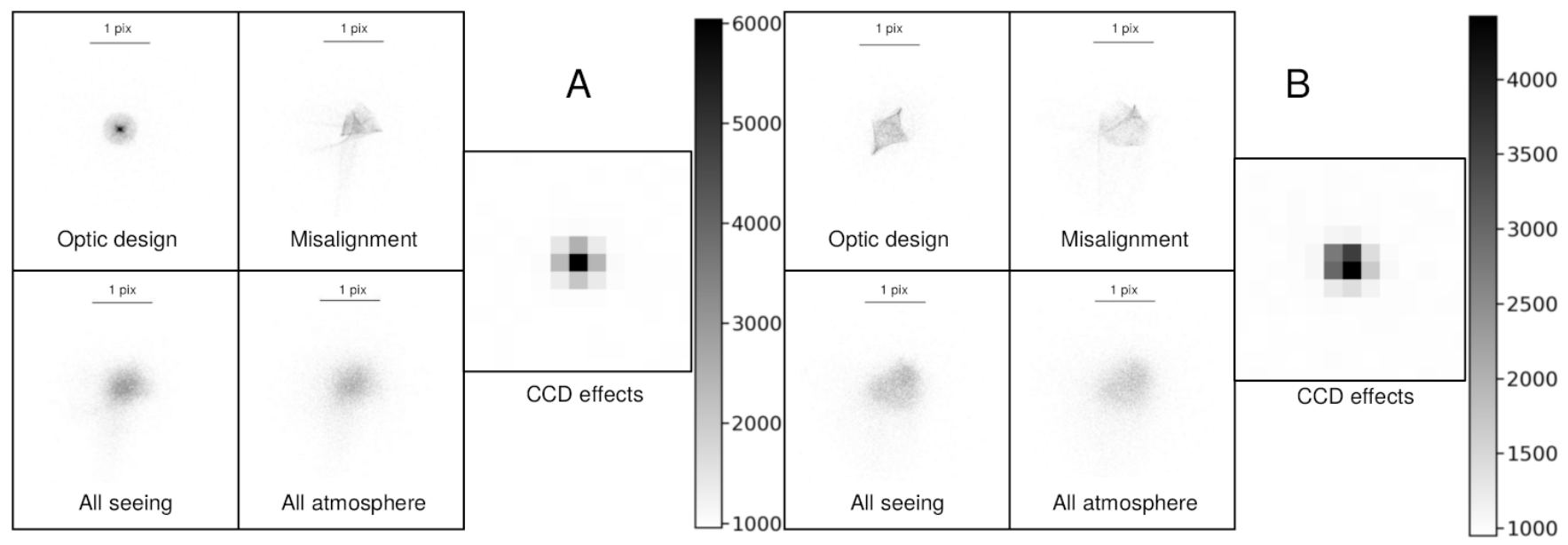

Figure 2. A: Photon Monte Carlo simulation of a star in the centre of the field of view, with PhoSim. The different simulation effects are added one after the other as follows. Upper left: optical design only. Upper right: perturbations, which include misalignment of surfaces and dust deposition. Lower left: seeing and dome seeing effects. Lower right: all atmospheric effects, including extinction, diffraction and clouds attenuation. Middle right: CCD image, including noise and gain conversion (values of counts are in ADU). B: same as before but with the star observed at the corner of the field of view, at a distance of $0.7 \times 1.2$ from the centre.

coat the cryostat window with a $g$-band LSST-like filter, at $\sim 471.1 \mathrm{~nm}$ and with a bandwidth of $\sim 123 \mathrm{~nm}$. The window has a thickness of $5 \mathrm{~mm}$ and it is made of silica.

A very important aspect to consider in the design of the pathfinder is the anti reflection (AR) coating to apply on the CCD. The typical AR coatings available for CCDs have lower transmission than the AR coatings used for lenses and often they let $>5 \%$ of the light to be reflected back. This means that for a classical Schmidt telescope design (onaxis and with the corrector plate) a large fraction of light is reflected back through the cryostat window and/or corrector plate and part of this will in turn be reflected in the CCD direction again, creating an unfocused image of the object observed. These ghost images limit the observable highest magnitude and prevents to reach the required level for ultralow surface brightness objects observation.

The pathfinder design is not immune to these features as the light reflected back from the CCD can pass trough the obscuration in the secondary mirror and be focused on the focal plane again by the spherical tertiary mirror. However we can greatly limit this effect by choosing an appropriate AR coating for the CCD. For the purpose of the simulation we selected the same AR coating used for the MUSE CCDs (Reiss et al. 2012) which are e2v CCD231-84, and their quantum efficiency $(\mathrm{QE})$ with reflectivity values $\sim \mathbf{1 . 2 - 2 . 0 \%}$ around the central wavelength of the filter. The noise properties, such as the readout and dark current, have been selected using datasheet of CCD290-99 from Teledyne-E2 ${ }^{4}$ and are listed in Table 2.

The impact of the curving process on these characteristics of the detectors has not been fully determined yet, while some manufacturer tested few prototypes and found increased values for the dark current (Gregory et al. 2015),

4 http://www.e2v.com/resources/account/

download-datasheet/1897
Table 2. CCD parameters used in the simulation.

\begin{tabular}{lr}
\hline \multicolumn{2}{c}{ CCD properties } \\
\hline Pixel pitch & $10 \mu \mathrm{m} / \mathbf{2}^{\prime \prime} \mathbf{3 2}$ on sky \\
Pixel number & $4030 \times 2480$ \\
Readout noise & $2.5 \mathrm{e}^{-}$ \\
Dark current & $4 \mathrm{e}^{-}$pixel $^{-1}$ hour $^{-1}$ \\
Gain & $1.7 \mathrm{e}^{-} \mathrm{ADU}^{-1}$ \\
Radius of curvature & $887.1 \mathrm{~mm}$ \\
Shape & convex \\
\hline
\end{tabular}

others found no clear performance degradation with respect to the flat sensor case (Lombardo et al. 2019).

\section{SIMULATED POINT SPREAD FUNCTION AND OBSERVED FIELD}

The quality of the PSF of the telescope is a key aspect to consider in ultra-low surface brightness objects observations, as already discussed in previous publications (Abraham \& van Dokkum 2014). The telescope must provide PSF whose wings are as low as possible, such that the faint emission of these objects is not dominated by the PSF residuals of brighter stars or galaxies in the same observed field.

\subsection{Point Spread Function from single star simulation}

The PSF of the pathfinder is shown in Figure 3 as function of the radial distance in arcmin. This PSF is obtained from a simulation of a 9 mag star in the centre of the field of view and it includes all the effects of optical design perturbations, atmosphere and seeing (described in Section 3). The PSF is 


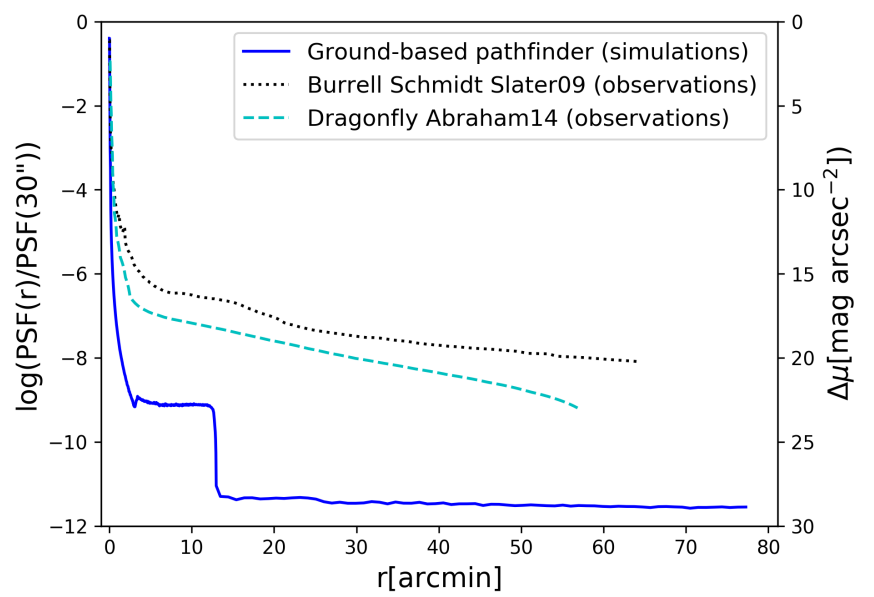

Figure 3. PSF of the ground-based pathfinder presented in this paper from photo Monte Carlo simulations in solid blue line, PSF of Dragonfly in cyan dashed line (Abraham \& van Dokkum 2014) and PSF of the Burrell Schmidt in dotted black line (Slater et al. 2009). All curves are normalized with their values within $30^{\prime \prime}$.

computed from an image composed of 140 different observations where the CCD was exposed for $40.5 \mathrm{~s}$ each time. For each of the images, we allowed the atmospheric parameters to variate randomly in the simulations. Additionally, the simulation does not include any CCD effects as we do not want to be limited by any systematic effect for our PSF estimation (e.g., the saturation of the central pixel).

Each value of the PSF in Figure 3 is an average of the number of photons at the focal plane that are inside the pixels located at the same distance from the centre of the star (for each ring). The two additional curves in Figure 3 show the PSFs for Dragonfly and the Burrell Schmidt telescope (respectively Abraham \& van Dokkum 2014; Slater et al. 2009). All curves are normalized within $30^{\prime \prime}$ from the centre, and we can see how low the PSF wings are for the pathfinder. This comparison can be used only as a qualitative estimation of the improvements achievable with a curved focal plane design optimized for ultra-low surface brightness observations, as the data for the pathfinder are purely extracted from simulations whereas the other two are estimates from real observations and hence include more systematic effects.

The PSF of the pathfinder appears to decrease rapidly down to $10^{-9}$, where it plateaus. This plateau extends from a distance of 3' to 13' from the centre of the field of view, and it is caused by a ghost image of the star due to the back reflection from CCD and filter. The ghost, however, does not degrade the PSF as it is suppressed by 8 orders of magnitudes with respect to the centre. After 13' the PSF decreases again reaching the $10^{-11.5}$ level.

A $2 \mathrm{D}$ view of the PSF is shown in Figure 4 (left image), where every square represents a pixel of the detector in the focal surface of the telescope. From the Figure we can distinguish the black central area, where most counts are, (67\% of the total number of photons are inside the central pixel and $98 \%$ are within a circle of 5.' 8 radius) and the large plateau that extends up to $13^{\prime}$. Some of the photons simulated do not reach the focal plane at all as they first hit the primary mirror of the telescope with large angles and end up scattered outside immediately or after a few bounces on the other two mirrors of the telescope.

In Figure 4 we can also find the 2D image of the PSF for the star observed at $0.7 \times 1.2$ from the centre of the field of view (right image). The PSF appears almost identical to the previous case $(65 \%$ of the total number of photons are inside the central pixel and $98 \%$ are within a circle of $5 . \prime 8$ radius), with only the ghost image shifted with respect to the centre of the PSF. A better comparison between these two cases is shown in Figure 5 where we can see that the two curves overlap. Since in Figure 4 (right image) and Figure 5 the observed star is located at the corner of the field of view, where the highest amount of image deformation is expected, we can conclude that the PSF of the pathfinder is uniform across the full field of view.

\subsection{Simulation of a field in the sky}

The main science goal of the pathfinder is the observation of ultra-low surface brightness objects. In order to test our capacity to observe such faint emissions, we simulate the entire telescope system in the case of an observational campaign of a sky field of $5^{\prime} \times 5^{\prime}$, this time also including the CCD effects.

The field is composed of stars and galaxies drawn from the Millennium Simulation and generated using CatSim ${ }^{5}$. In addition to this, a large elliptical galaxy (of 2.'5 observed angular diameter) and an arch-like structure have been added to the simulation at the centre of the field. The galaxy has an integrated brightness of $13.5 \mathrm{mag}$ and the arches have surface brightness of $29 \mathrm{mag} \operatorname{arcsec}^{-2}$ which makes the full image similar to NGC5907 (Martínez-Delgado et al. 2010).

In the simulations the field is observed at an airmass value of 1.13 , for a total time of $\sim 30$ hours, which is equivalent to a week of observations in point-and-stare mode. The observation is carried out through a series of short exposures of $382 \mathrm{~s}(\sim 6 \mathrm{~min})$ and summed together to increase the contrast. This exposure time is equivalent to the passage of the star across the full sensor in a hypothetical drift-scan observation.

For each of these simulations a new set of atmospheric parameters and (this time also) CCD effects were used. Figure 6 shows the distribution of the parameters that mostly influence the atmospheric variation for the 288 exposures considered: for the seeing we have a mean value of $1{ }^{\prime \prime} 02 \pm 0 . \prime 06$, the median cloud extinction is $0.15 \mathrm{mag}$, the average aerosol optical depth has a value of $0.061 \pm 0.010$ and the wind speed is in average $6.2 \pm 3.3 \mathrm{~m} \mathrm{~s}^{-1}$.

When we compare these values to the variations found within a year (excluding the months of June. July and August, which are the ones with the highest background emission in La Palma) at the Observatorio delRoque de los Muchachos in La Palma ${ }^{6}$, we find that the simulated variations are smaller than the observed ones. The seeing shows standard deviations between $0 . ' 2$ and $0 . ' 3$, while the average value is around $\sim 0^{\prime \prime} 93$. This means that the simulations are considering a more stable worst seeing condition with

\footnotetext{
5 https://www.lsst.org/scientists/simulations/catsim

6 http://www.ing.iac.es/astronomy/observing/conditions/
} 

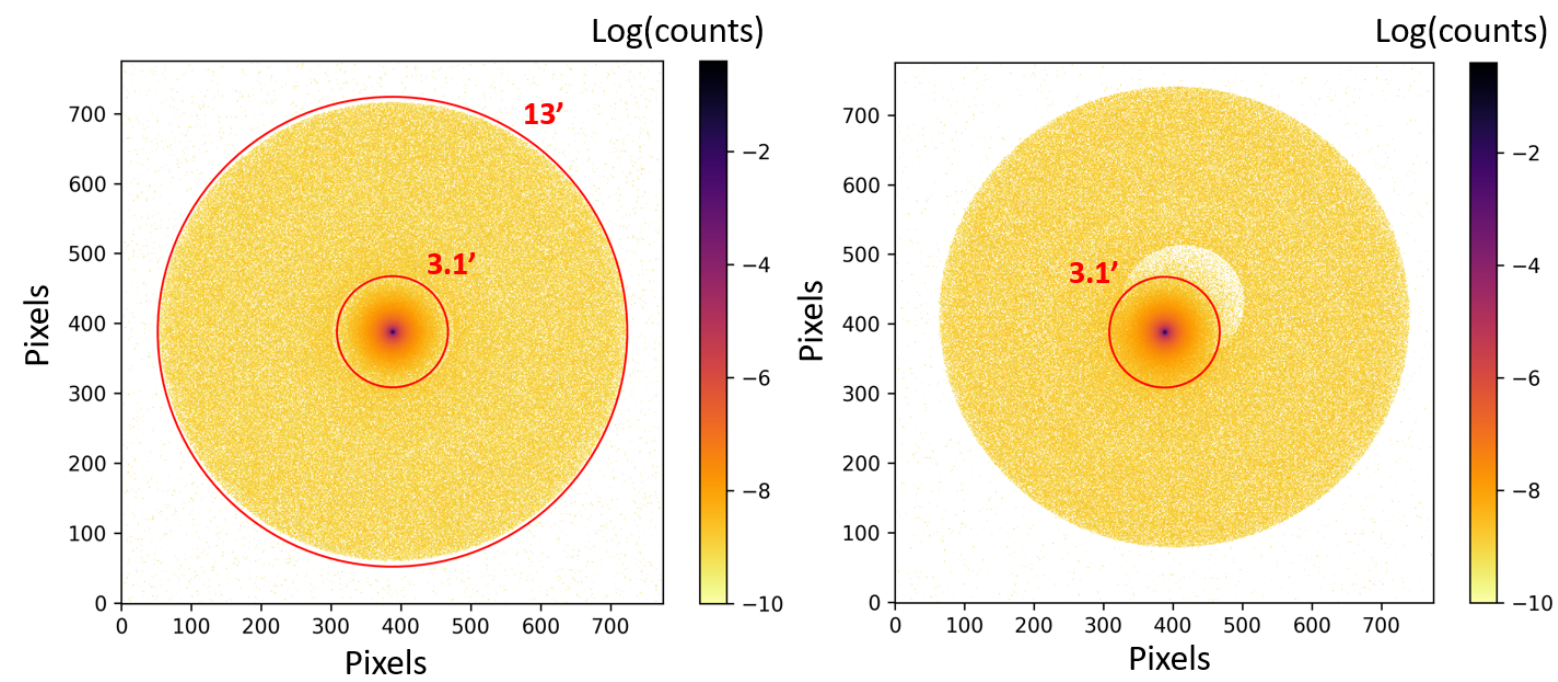

Figure 4. 2D view of the PSF of the pathfinder shown in Figure 3 for a star observed at the centre of the field of view (left) and at a distance of $0.7 \times 1.2$ from the centre (right). The red circles represents radial distances of $3 .^{\prime} 1$ and $13^{\prime}$.

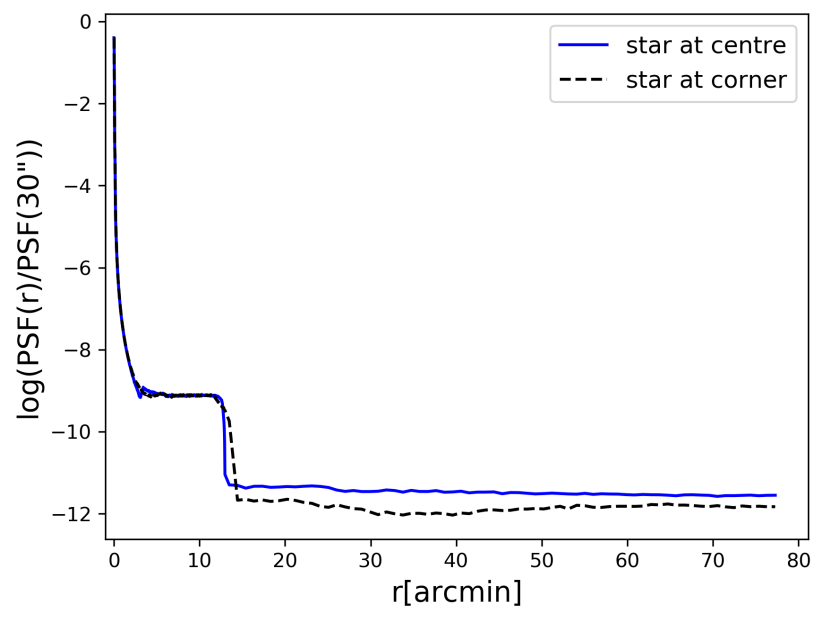

Figure 5. PSF of the ground-based pathfinder from photo Monte Carlo simulations of a star at the centre of the field of view of the pathfinder (blue full line) and at the corner $(0.7,1.2)$ (dashed black line). All curves are normalized with their values within $30^{\prime \prime}$.

respect to what has been observed nearby the observatory. Regarding the aerosol values measured at the Izaña location during the year 2017, they present variations of 0.2 against the 0.1 of the simulations. Even if there is a discrepancy, we can still say that the atmospheric parameters in the simulations well represent the variability of the observing conditions in a 9 months period.

The results of the simulations of the $5^{\prime} \times 5^{\prime}$ field observed at the centre of the field of view of the pathfinder are shown in Figure 7. At the top image a single frame of $6 \mathrm{~min}$ of integration is plotted, which corresponds to one night observation of drift-scan. Once the image is integrated for several consecutive nights (until it reaches $45 \mathrm{~min}$ of integration), the field appears more populated and better defined (middle image). It is possible to observe also the faint arch-like structure around the bright galaxy in the middle. This image is equivalent to a week of observations in drift-scan mode.

If one continues to integrate until the total exposure time is $30 \mathrm{~h}$, corresponding to $\sim 10$ months of drift-scan or 1 week of point-and-star observation mode, more objects appear and the arch-like structure is more visible. In the drift-scan case the $\sim 10$ months represent the total number of days required for the observations, and it will be spread over a few years depending on the specific object visibility in the sky.

The signal to noise ratios $(\mathrm{S} / \mathrm{N})$ obtained per pixel in the ULSB object is $\sim 29, \sim 7, \sim 4$, for the 30 $\mathrm{h}, 45 \mathrm{~min}$ and $6 \mathrm{~min}$ integration time images respectively. These are computed by running again the simulations with only the ULSB object in the field with the same atmospheric conditions used for the full field simulations. The median signal across the pixels composing this ULSB object is, then, extracted and divided by the square root of the median signal of the ULSB object from the full field image (which contains not only the faint object but also the noise and the background noise due to the presence of background stars and galaxies). The pixels of the ULSB object that overlap with the bright stars in the full field simulations are excluded from both calculations. These results show that we are able to observe, in a fraction of sky equivalent to 1 pixel $\left(5.4 \operatorname{arcsec}^{2}\right)$, a feature with surface brightness of 29 mag $\operatorname{arcsec}^{-2}$ at a precision of $\sim 3 \%, \sim 13 \%$ or $\sim 26 \%$ depending on the exposure time used.

The presence of the ULSB object in the images implies the capability of the pathfinder of observing such extremely faint and extended objects and of reaching a good $\mathbf{S} / \mathbf{N}$, even after just one week of nightly integration time. 

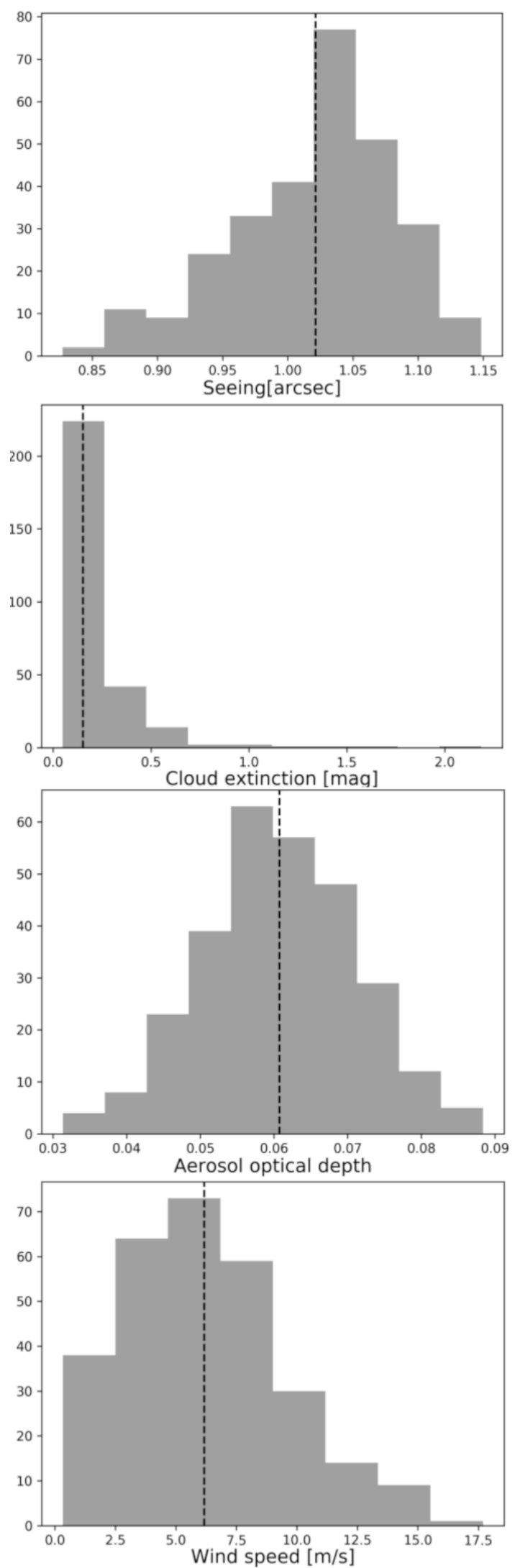

Figure 6. Histograms of the atmospheric parameter variations for the 288 shorter exposures that compose the 30 hours observation of the simulated $5^{\prime} \times 5^{\prime}$ field. The vertical lines show the position of the mean value of the distributions. $\log ($ counts (ADU))
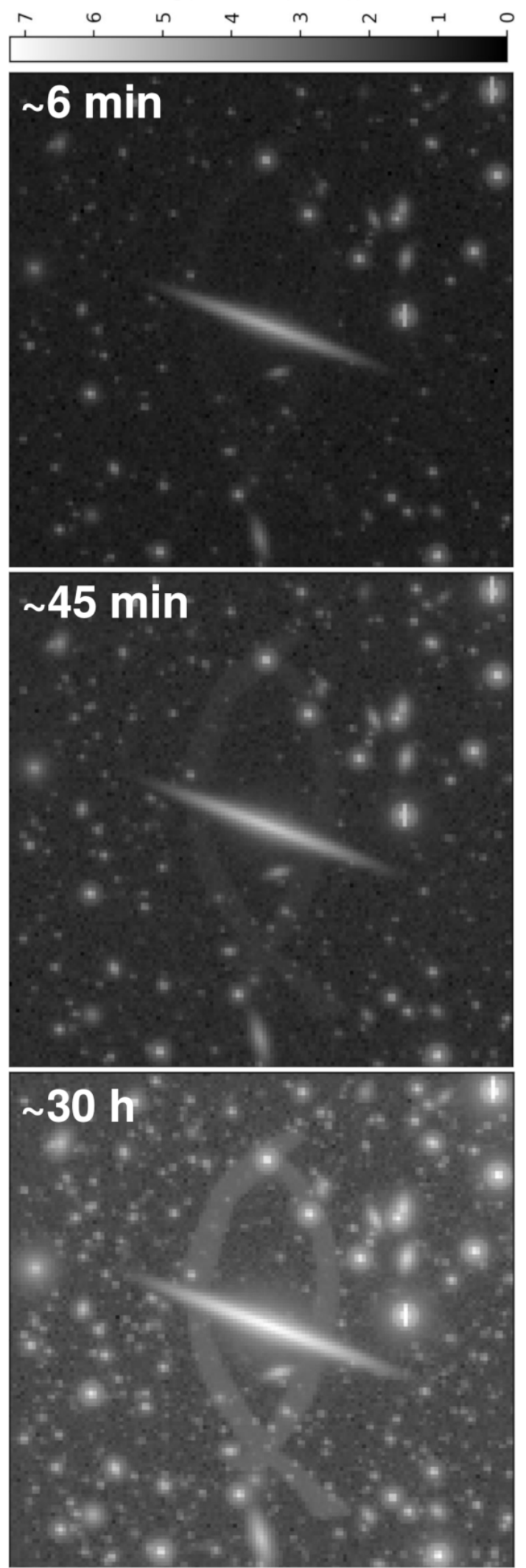

Figure 7. Simulation of a $5^{\prime} \times 5^{\prime}$ field observed by the groundbased pathfinder after an exposure time of 6 min (top image), 45 min (middle image) and $30 \mathrm{~h}$ (bottom image). 


\section{CONCLUSIONS}

The ultra-low surface brightness universe still remains a niche for observations, as a full-sky survey is missing. As observations of this kind demand a telescope with an highly optimized design, we propose here an alternative concept that uses a curved detector. The advantage of such telescope is due to its unique design that combines the wide field of view of a Schmidt telescope, with the compactness, the lack of supporting spiders and field flattening optics provided by the usage of a curved CCD to match its curved focal surface. All these elements contribute to have a Point Spread Function with very low wings, key element for the observation of the ultra-faint universe.

The proposed telescope is a fully reflective, off-axis Schmidt design, with an anamorphic primary mirror of $35.6 \mathrm{~cm}$ diameter, a flat secondary mirror and a spherical tertiary mirror that focuses the light onto a convex sphericallycurved CCD. In this paper we tested the full system through full scale photon Monte Carlo simulations, with the software PhoSim. This software allows to simulate all the effects due to Earth atmosphere, the effects of misalignment of the optics and the propagation of the photon in the CCD, including the noise effect of the detector itself.

These full scale simulations are the only way to study the illumination distribution of the full focal plane of the telescope and are mandatory to ascertain the potentiality of the pathfinder and the capability to reach the extremely low brightness level in the observations. The results from the simulation of a star at the centre and at the corner of the field of view have shown firstly that the PSF is uniform across the full field of view, and secondly that the wings of the PSF reach unprecedentedly low level. The normalised PSF decreases down to $10^{-11.5}$ at a radial distance from its centre of $\sim 13^{\prime}$ which correspond to a difference in mag $\operatorname{arcsec}^{-2}$ of 27 with respect to its value at the centre. Before reaching these values, the PSF shows a plateau of $\sim 10^{-9}$ due to the ghost image of the star itself. The light of the star is in fact reflected back from the CCD and the filter and than it is propagated again in the direction of the focal surface reaching the CCD surface in a displaced position. This unfocused image of the star creates an halo of photons that extends from $\sim 3^{\prime}$ to $\sim 13^{\prime}$ 'from the centre of the PSF. The PSF quality is not degraded by the presence of such ghost, as it is suppressed by 8 orders of magnitude with respect to the PSF central value.

Finally we simulated a field of galaxies and stars of $5^{\prime} \times 5^{\prime}$ observed at the centre of the field of view for a total exposure time of 30 hours. This exposure time is reached by adding up 288 shorter observations of $382 \mathrm{~s}(\sim 6 \mathrm{~min})$ and for each of these sub-exposures the atmospheric conditions have been changed randomly. The final image clearly shows the presence of the extended structure injected in the simulation and that is faintly emitting at $29 \mathrm{mag} / \operatorname{arcsec}^{2}$, typical brightness for the ultra-low surface brightness objects. These results illustrate the full potentiality of the pathfinder, that will not only be used to test the groundbreaking technology of the curved sensors, but also provide important science outcome.

The design concept shown in this paper can, hence, serve as alternative to all current surveys for ULSB observations, or more in general targeted to extended objects. The installation of more telescopes of this kind could in principle extend the current field of view and photon collection capability and could provide a better sampling of the sky, especially if located also in the Southern hemisphere.

\section{ACKNOWLEDGEMENTS}

The authors would like to thank David Valls-Gabaud for his contribution in the project, and the referee for the constructive comments that improved the manuscript. The authors acknowledge the support of the European Research council through the H2020 - ERC-STG2015 - 678777 ICARUS program. This activity was partially funded by the French Research Agency (ANR) through the LabEx FOCUS ANR-11-LABX-0013.

\section{REFERENCES}

Abraham R. G., van Dokkum P. G., 2014, PASP, 126, 55

Atkinson A. M., Abraham R. G., Ferguson A. M. N., 2013, ApJ, 765,28

Barton I. J., Thompson L. A., 1997, AJ, 114, 655

Blake T., et al., 2013, in Advanced Maui Optical and Space Surveillance Technologies Conference. p. E19

Boissier S., et al., 2008, ApJ, 681, 244

Bullock J. S., Boylan-Kolchin M., 2017, ARA\&A, 55, 343

Cooper A. P., et al., 2010, MNRAS, 406, 744

Cooper A. P., D'Souza R., Kauffmann G., Wang J., BoylanKolchin M., Guo Q., Frenk C. S., White S. D. M., 2013, MNRAS, 434, 3348

Duc P.-A., et al., 2015, MNRAS, 446, 120

Fry A. M., Morrison H. L., Harding P., Boroson T. A., 1999, AJ, 118, 1209

García-Gil A., Muñoz-Tuñón C., Varela A. M., 2010, PASP, 122, 1109

Gregory J. A., et al., 2015, Applied Optics, 54, 3072

Guenter B., et al., 2017, Optics Express, 25, 13010

Hugot E., Wang X., Valls-Gabaud D., Lemaître G., Agócs T., Shu R., Wang J., 2014, in Space Telescopes and Instrumentation 2014: Optical, Infrared, and Millimeter Wave. p. 91434X, doi:10.1117/12.2057461

Kalnay E., et al., 1996, Bull. Amer. Meteorolog. Soc., 77, 437

Kazantzidis S., Bullock J. S., Zentner A. R., Kravtsov A. V., Moustakas L. A., 2008, ApJ, 688, 254

Knapen J. H., Trujillo I., 2017, in Knapen J. H., Lee J. C., Gil de Paz A., eds, Astrophysics and Space Science Library Vol. 434, Outskirts of Galaxies. p. 255 (arXiv: 1612.05405), doi:10.1007/978-3-319-56570-5_8

Leinert C., Vaisanen P., Mattila K., Lehtinen K., 1995, A\&AS, 112,99

Lombardo S., et al., 2019, Appl. Opt., 58, 2174

Martínez-Delgado D., et al., 2010, AJ, 140, 962

Mihos J. C., Harding P., Feldmeier J. J., Rudick C., Janowiecki S., Morrison H., Slater C., Watkins A., 2017, ApJ, 834, 16

Muslimov E., Hugot E., Jahn W., Vives S., Ferrari M., Chambion B., Henry D., Gaschet C., 2017a, Optics Express, 25, 14598

Muslimov E., et al., 2017b, Appl. Opt., 56, 8639

Olivier S. S., Seppala L., Gilmore K., 2008, in Advanced Optical and Mechanical Technologies in Telescopes and Instrumentation. p. $70182 \mathrm{G}$, doi:10.1117/12.790264

Peterson J. R., et al., 2015, ApJS, 218, 14

Reiss R., Deiries S., Lizon J.-L., Rupprecht G., 2012, in Groundbased and Airborne Instrumentation for Astronomy IV. p. 84462P, doi:10.1117/12.925388 
Rim S.-B., Catrysse P. B., Dinyari R., Huang K., Peumans P., 2008, Optics Express, 16, 4965

Singaravelu B., Cabanac R. A., 2014, PASP, 126, 386

Slater C. T., Harding P., Mihos J. C., 2009, PASP, 121, 1267

Valls-Gabaud D., MESSIER Collaboration 2017, in Gil de Paz

A., Knapen J. H., Lee J. C., eds, IAU Symposium Vol. 321, Formation and Evolution of Galaxy Outskirts. pp 199-201, doi:10.1017/S1743921316011388

Zaritsky D., Schectman S. A., Bredthauer G., 1996, PASP, 108, 104

van Dokkum P. G., Abraham R., Merritt A., Zhang J., Geha M., Conroy C., 2015, ApJ, 798, L45

This paper has been typeset from a $\mathrm{T}_{\mathrm{E}} \mathrm{X} / \mathrm{LATE} \mathrm{X}$ file prepared by the author. 